\title{
Application of Jasmonic Acid Followed by Salicylic Acid Inhibits Cucumber mosaic virus Replication
}

\author{
Ying Luo ${ }^{1,}$, Jing Shang ${ }^{2,}$, Pingping $\mathrm{Zhao}^{2}$, Dehui $\mathrm{Xi}^{2}$, Shu Yuan ${ }^{2}$ and Honghui Lin ${ }^{2,3 *}$ \\ ${ }^{1}$ College of Life Science and Biotechnology, Mianyang Normal University, Mianyang 621000, Sichuan, China \\ ${ }^{2}$ Key Laboratory of Bio-resources and Eco-environment (Ministry of Education), Sichuan University, Chengdu 610064, China \\ ${ }^{3}$ State Key Laboratory of Hydraulics and Mountain River Engineering, Sichuan University, Chengdu 610065, China \\ (Received on November 16, 2010; Accepted on December 16, 2010)
}

\begin{abstract}
Systemic acquired resistance is a form of inducible resistance that is triggered in systemic healthy tissues of local-infected plants. Several candidate signaling molecules emerged in the past two years, including the methylated derivatives of well-known defense hormones salicylic acid (SA) and jasmonic acid (JA). In our present study, the symptom on Cucumber mosaic virus (CMV) infected Arabidopsis leaves in $0.1 \mathrm{mM}$ SA or $0.06 \mathrm{mM}$ JA pre-treated plants was lighter (less reactive oxygen species accumulation and less oxidative damages) than that of the control group. JA followed by SA $(\mathrm{JA} \rightarrow \mathrm{SA})$ had the highest inhibitory efficiency to CMV replication, higher than $J A$ and $S A$ simultaneous co-pretreatment (JA+SA), and higher than a JA or a SA single pretreatment. The crosstalk between the two hormones was further investigated at the transcriptional levels of pathogenesis-related genes. The time-course measurement showed JA might play a more important role in the interaction between $\mathrm{JA}$ and $\mathrm{SA}$.
\end{abstract}

Keywords : Jasmonic acid, plant immune, salicylic acid, virus control

Salicylic acid (SA) and jasmonic acid (JA) are important signaling molecules in plant defense responses. The contribution of these signaling molecules in plant defense differs depending on the invading pathogen. For example, the SAmediated defences have a major role in the basal resistance to the bacterial and Oomycete pathogens, Pseudomonas syringae and Peronospora parasitica, respectively, and also Turnip crinkle virus (TCV) and Cucumber mosaic virus (CMV) (Dong, 2001; Feys and Parker, 2000; Kachroo et al., 2000; Pieterse et al., 2001; Takahashi et al., 2002). In contrast, JA signaling has an important role in the basal resistance to the fungal pathogen, such as Botrytis cinerea

\footnotetext{
*Corresponding author.

Phone) +86-28-85415389, FAX) +86-28-85415300

E-mail)honghuilin@hotmail.com

$\S$ equally contributed.
}

(Thomma et al., 1998). SA-and JA-dependent defense pathways have been shown to cross-communicate providing the plant with a regulatory potential to finetune the defense reaction depending on the type of attacker encountered. Induced resistance is regulated by a network of interconnecting signal transduction pathways in which SA and JA function as key signaling molecules (Glazebrook, 2001; Pieterse et al., 1998; Reymond and Farmer, 1998; Thomma et al., 2001).

Our previous study showed that application of $0.06 \mathrm{mM}$ JA and then of $0.1 \mathrm{mM}$ SA $24 \mathrm{~h}$ later, enhanced Arabidopsis, tobacco, tomato and hot pepper resistance to $\mathrm{CMV}$, tobacco resisitance to Tobacco mosaic virus, and tobacco and tomato and hot pepper resisitance to TCV. The inhibition efficiency to virus replication would usually achieve up to $80-90 \%$ (Shang et al., 2011). However, the putative molecular mechanism was not fully investigated. In this study, time-course expressions of SA/JA-related genes were detected. The measurement showed that JA may play a more important role than SA in this phytohormone interaction.

\section{Materials and Methods}

Plant culture and pathogen inoculation. All plants were grown in a temperature-controlled growth chamber with a 16 h-light 8 h-dark cycles at $20-25^{\circ} \mathrm{C}$. The inoculation with virus was carried out as described previously (Shang et al., 2009). In brief, Carborundum was evenly applied to the surface of leaves, and virus, at a concentration of $1 \mu \mathrm{g} / \mathrm{ml}$ in $10 \mathrm{mM}$ HEPES buffer $\mathrm{pH}$ 7.0, was rubbed onto the leaves with cheese cloth.

Before $24 \mathrm{~h}$ of virus inoculation, the seedlings were sprayed with JA (Sigma, St. Louis, MO, USA; methyl jasmonate could also be used and with the same concentration) followed by SA (Sigma). The optimal concentrations of JA and SA were 0.06 and $0.1 \mathrm{mM}$, respectively, and the optimal time for SA was $24 \mathrm{~h}$ after JA. 
RNA analysis. Total RNAs were isolated from plant tissues by Trizol reagent (Invitrogen). The RNAs were separated on $1.0 \%$ denaturing agarose gels. For RT-PCR, the first strand cDNA was prepared using the ReverTra Ace kit (TOYOBO). To further assay the expression levels of gene, quantitative real-time PCR analysis was performed on a Bio-Rad iCycler. CMV-CP was amplied with degenerate primers CMV-F (5'-CTGAACGGGTTGTCCATC3') and CMV-R (5'-TGGTCTCCTTTWRGAGRC-3', R= AorG; W=AorT). PRI was amplied with primers PR1-F (5'-TCTTTGTAGCTCTTGTAGGTG-3') and PR1-R (5'GATTCTCGTAATCTCAGCTCT-3'). PR2 was amplied with primers PR2-F (5'-CTCTTTACAAACAACAAAAC3') and PR2-R (5'-TTCATACTTAGACTGTCGAT-3'). NPR1 was amplied with primers NPR1-F (5'-GATTCGGTTGTGACTGTTTTGG-3') and NPR1-R (5'-TCTCGTTTGTCTTCTTGCTCTA-3'). WRKY53 was amplied with primers WRKY53-F (5'-CATCGCCAAGATTACATT-3') and WRKY53R (5'-AGTCTGCGGCTACTACCA-3'). WRKY70 was amplied with primers WRKY70-F (5'-AAAAGATTGGGACCCGTTAA-3') and WRKY70-R (5'-TGGGAGTTTCTGCGTTGG-3'). GST was amplied with primers GST-F (5'AGACTTTGGAGGGACGAG-3') and GST-R (5'-ACGGAGGACTACAAGAACTA-3'). PDF1.2 was amplied with primers PDF1.2-F (5'-GCTAAGTTTGCTTCCATCAT-3') and PDF1.2-R (5'-CATGGGACGTAACAGATACAC-3'). MPK4 was amplied with primers MPK4-F (5'-TATTCGCTCTAACCAACC-3v) and MPK4-R (5'-TCAACTGTGATGCGTCTG-3'). The cDNA was amplified by using SYBR Premix Ex Taq (TaKaRa). The amplification of the target genes was monitored every cycle by SYBR-green I fluorescence. The $\mathrm{Ct}$ (threshold cycle), defined as the PCR cycle at which a statistically significant increase of reporter fluorescence was first detected, was used as a measure for the starting copy numbers of the target gene. Relative quantitation of the target gene expression level was performed using the comparative $\mathrm{Ct}$ method. Three technical replicates were performed for each experiment. Actinl gene was used as an internal control (F primer: 5'-CATCAGGAAGGACTTGTACGG-3'; R primer: 5'-GATGGACCTGACTCGTCATAC-3').

Oxidative damage estimation. The $\mathrm{H}_{2} \mathrm{O}_{2}$ content of leaves was measured as described by Velikova et al. (2000). Approx. $0.5 \mathrm{~g}$ of fresh leaves were cut into small pieces and homogenized in an ice bath with $5 \mathrm{~mL} 0.1 \%(\mathrm{w} / \mathrm{v})$ trichloroacetic acid (TCA). The homogenate was centrifuged at $12,000 \times \mathrm{g}$ for $20 \mathrm{~min}$ at $4^{\circ} \mathrm{C} .0 .5 \mathrm{~mL}$ of the supernatant was added to $0.5 \mathrm{~mL} 10 \mathrm{mM}$ potassium phosphate buffer ( $\mathrm{pH} 7.0$ ) and $1 \mathrm{~mL} 1 \mathrm{M} \mathrm{KI}$. The absorbance of the supernatant was read at $390 \mathrm{~nm}$.

Lipid peroxidation was estimated by measuring the thio- barbituric acid-reactive substances (TBARS) as previously described (Xi et al., 2007). The lipid peroxides were expressed as TBARS content.

Electrolyte leakage was measured according to Cao et al. (2009). After measuring the conductivity, the Arabidopsis leaves samples were boiled for $15 \mathrm{~min}$ to achieve $100 \%$ electrolyte leakage.

Superoxide and $\mathrm{H}_{2} \mathrm{O}_{2}$ staining for in situ detection. In situ superoxide $\mathrm{H}_{2} \mathrm{O}_{2}$ were detected with nitroblue tetrazolium (NBT) and 3,3-diaminobenzidine (DAB), respectively, as described previously (Shang et al., 2010). Arabidopsis leaves were excised at the base with a razor blade and supplied through the cut ends with NBT (1 mg/ $\mathrm{mL})$ or DAB $(0.5 \mathrm{mg} / \mathrm{mL})$ solutions for $8 \mathrm{~h}$. Leaves were then decolourized in boiling ethanol (95\%) for $15 \mathrm{~min}$.

\section{Results}

The virus replication of Arabidopsis leaves infected with CMV. In the present study, Arabidopsis leaves were pretreated with $0.1 \mathrm{mM} \mathrm{SA}$ and $0.06 \mathrm{mM} \mathrm{JA}$. Fig. 1 showed the CMV-CP level days after CMV inoculation. JA followed by SA (JA $\rightarrow \mathrm{SA}$ ) had the highest inhibitory efficiency to CMV replication (over $80 \%$ ), higher than JA and SA simultaneous co-pretreatment $(\mathrm{JA}+\mathrm{SA})$, and than JA or SA single pretreatment. The expression levels of CMV-CP gene was further confirmed by the quantitative real-time PCR analysis (Fig. 1).

Superoxide and $\mathrm{H}_{2} \mathrm{O}_{2}$ staining. DAB staining confirmed the result that the lowest $\mathrm{H}_{2} \mathrm{O}_{2}$ accumulation occurred in

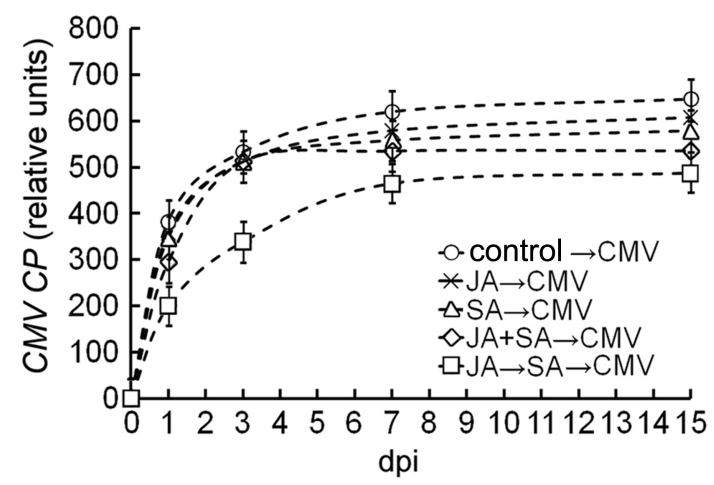

Fig. 1. CMV-CP gene expression levels of Arabidopsis systemic leaves infected with CMV. The CMV replication levels were detected by quantitative real-time PCR analysis at $0,1,3,7,15$ days post inoculation. "JA $\rightarrow$ SA" indicates $0.06 \mathrm{mM}$ JA followed by $0.1 \mathrm{mM} \mathrm{SA}$ pretreatment at the $24 \mathrm{~h}$ interval and before the virus inoculation at $24 \mathrm{~h}$; "J+S" means JA and SA simultaneous co-pretreatment; "SA" indicates SA pretreatment alone; "JA" indicates JA pretreatment alone. Error bars show standard deviations $(n=3)$. 


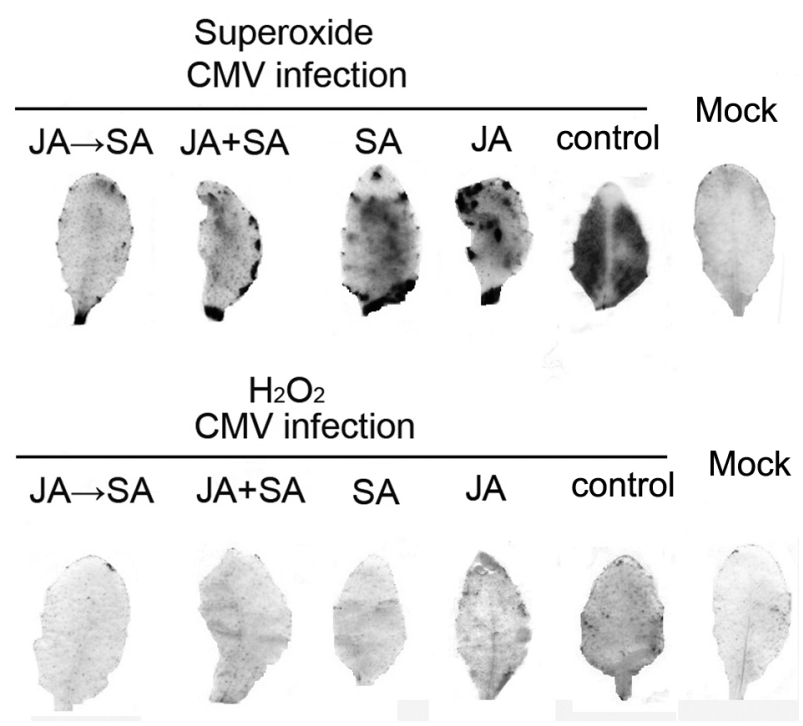

Fig. 2. DAB and NBT staining of the CMV-infected Arabidopsis leaves.

$\mathrm{JA} \rightarrow \mathrm{SA}$ pretreated leaves, and lower than that of JA/SA single treated leaves or that of JA+SA treated leaves (Fig. 2). Corresponding to this result, accumulation of superoxide (stained by NBT) in JA $\rightarrow$ SA pretreated leaves was the lowest, when compared with other treatments (Fig. 2). The results suggested that JA $\rightarrow$ SA treatment was the most effective to CMV resistance.

Degrees of membrane injury. TBARS are one of the most important lipid peroxidation products. The TBARS content reflects the degree of membrane injury, and is an important injury indicator of plants under stress. The TBARS of the JA followed by SA pretreatment $(\mathrm{JA} \rightarrow \mathrm{SA})$ was lighter than JA and SA simultaneous co-pretreatment (JA+SA), and than JA or SA single pretreatment (Fig. 3). $\mathrm{H}_{2} \mathrm{O}_{2}$ levels and electrolyte data reflected a similar trend (Fig. 3). The results suggest that virus infection reduces the stability and integrity of the plasma membrane, but the leaves pretreated with JA and SA were less affected.

Possible molecular pathways. The putative molecular pathways was investigated and some possible signaling factors have been defined. Transcripts of two transcription factors WRKY53 (Miao and Zentgraf, 2007) and WRKY70 (Li et al., 2004), MAP Kinase 4 (MPK4), JA-responsible resistance protein PDF1.2, SA-responsible resistance protein NPR1, PR1 and PR2 (Durrant and Dong, 2004; Spoel et al., 2003; Spoel et al., 2009) and ROS-related resistance protein glutathione S-transferase (GST) (Uquillas et al., 2004) were quantified in Arabidopsis seedlings inoculated with CMV (Fig. 4).

The expression level of NPRl inhibited by JA was de-

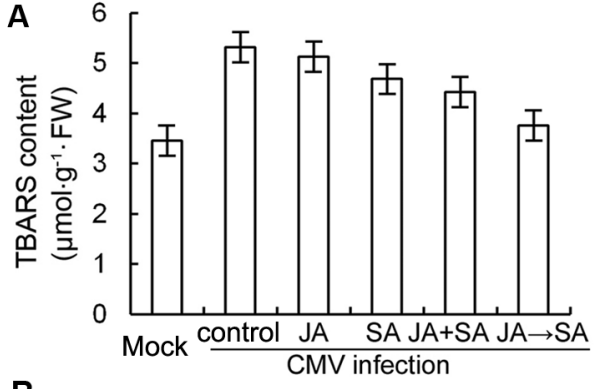

B

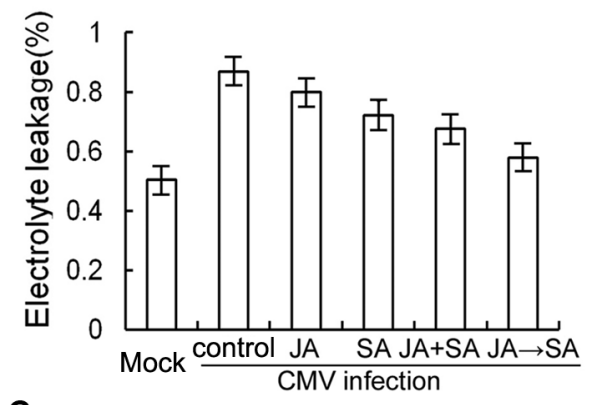

C

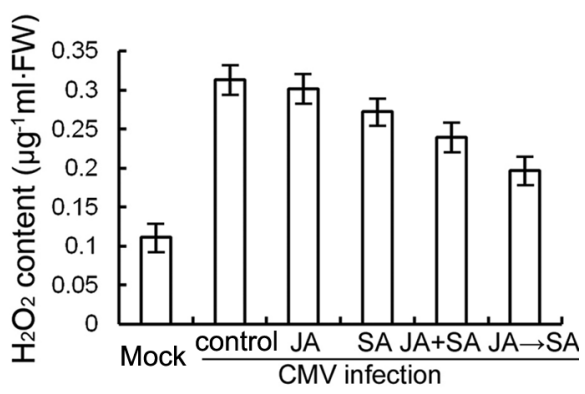

Fig. 3. Hydrogen peroxide, TBARS, and electrolyte leakage of the CMV-infected Arabidopsis leaves. Bars represent standard deviations of 3 independent replicates $(n=3)$.

creased first and then increased. On the contrary, the expression level of NPRI was promoted by SA, and the level was gradually increased. For two hormones simultaneous co-treatment (JA+SA), in the case that SA works before JA, $N P R 1$ expression increased first and then decreased. For JA followed by SA treatment (JA $\rightarrow \mathrm{SA}$ ), JA works before $\mathrm{SA}$, and the expression level of NPRI decreased and then increased to a relatively stable level. It was maintained at a suitable level at $15 \mathrm{dpi}$, but not increase dramatically during the infection. For CMV pure infection, the peak appeared at $7 \mathrm{dpi}$ and its expression increase dramatically.

The expression level of $P R 1$ was inhibited by JA, which decreased first and then increased. But the expression level of $P R 1$ was promoted by SA and was gradually increased then. The maximum peak appeared at 1 dpi. For JA+SA, in the case that SA works before JA, the expression level of PRI therefore increased first and then decreased. The peak appeared at $3 \mathrm{dpi}$. For JA $\rightarrow$ SA, JA works before SA, and the expression level of $P R 1$ was decreased first and then 

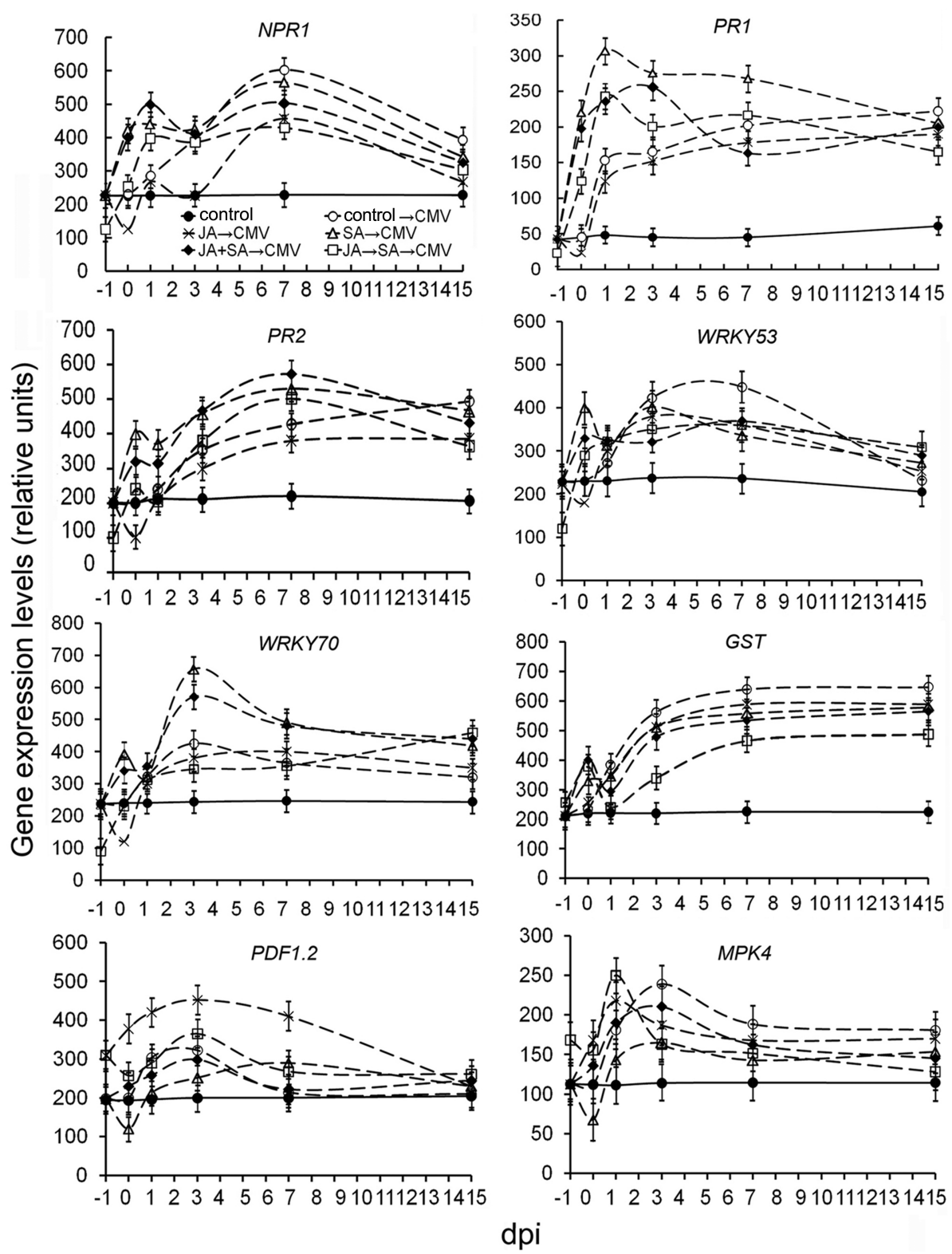

Fig. 4. Defense gene expression levels in wild-type Arabidopsis seedlings were detected by quantitative real-time PCR analysis at $-1,0$, $1,3,7$, and 15 days post inoculation (dpi). "JA $\rightarrow$ SA" indicates $0.06 \mathrm{mM} \mathrm{JA}$ followed by $0.1 \mathrm{mM}$ SA pretreatment at the $24-\mathrm{h}$ interval and before the virus inoculation at $24 \mathrm{~h}$; "J+S" means JA and SA simultaneous co-pretreatment; "SA" indicates solo SA pretreatment; "JA" indi-cates solo JA pretreatment. Error bars show standard deviations $(n=3)$.

increased. The peak appeared at 1 dpi. It was lower than single SA treatment or two hormones simultaneous cotreatment, but higher than the pure virus infection (without any treatment) or sprayed with JA. The expression level of PRI induced by SA but repressed by JA was the maker of activation of the SA-mediated defense response against viruses. That means JA-mediated defense response plays a leading role in JA $\rightarrow$ SA. Similarly, the expression level of $P R 2$ fluctuated like PR1. Differently, the peak appeared at 7 dpi.

The expression level of WRKY53 was inhibited by JA which was decreased first and then increased. By contrast, the expression of WRKY53 was promoted by SA which increased to the peak at $0 \mathrm{dpi}$ and then decreased. For $\mathrm{JA}+\mathrm{SA}$, in the case that SA works before JA, the expression level of WRKY53 was increased first and then decreased. For JA $\rightarrow \mathrm{SA}$, the expression level of WRKY53 was decreased and then increased which made its level relatively stable, while the gene expression of other treatments had larger fluctuations, showing more than one peaks. For CMV pure infection, the peak appeared at $7 \mathrm{dpi}$ and it shows only one peak. 
The change of $W R K Y 70$ expression level was similar to that of WRKY53. The only difference was that WRKY70 expression level rose to its peak at $3 \mathrm{dpi}$.

ROS contribute to the symptom development and pathogenesis in compatible plant-virus interactions, as described recently in plum pox virus (PPV) susceptible peach plants (Hernandez et al., 2006). The expression level of GST in pure virus inoculation was increased continuously. The expression level of GST was enhanced by spraying JA alone which was higher than SA single treatment. For $\mathrm{JA} \rightarrow \mathrm{SA}$, the GST expression level was the lowest. At $7 \mathrm{dpi}$, the gene exprssion level reached to the peak and then increased to a relatively stable level.

The expression of PDF1.2 was promoted by JA and the JA spraying alone made the level increased largest at $3 \mathrm{dpi}$. The expression was suppressed by spraying SA alone. For $\mathrm{JA}+\mathrm{SA}$, the peak appeared at $3 \mathrm{dpi}$, but was still lower than the JA $\rightarrow$ SA treatment.

The expression of MPK4 was promoted by JA. The peak appeared by spraying JA alone at $1 \mathrm{dpi}$. For JA $\rightarrow \mathrm{SA}$, the peak appeared at $1 \mathrm{dpi}$. The expression of MPK4 was inhibited by spraying SA. For JA+SA, the peak appeared at $3 \mathrm{dpi}$, the same as for pure virus infection.

Among these, WRKY53, WRKY70 and PDF1.2 showed the maximum expression in the JA $\rightarrow$ SA pretreated seedlings at $10 \mathrm{dpi}$, compared with the other treatments, therefore relating to the optimal effect of JA $\rightarrow$ SA. MPK4 transcript increased in all the seedlings inoculated with CMV. However, the increasing amplitude was the minimum in the $\mathrm{JA} \rightarrow$ SA pretreated seedlings, compared with the other treatments.

\section{Discussion}

JA is a potential lipid-derived systemic acquired resistance (SAR) signal which might be an early signal establishing systemic immunity (Truman et al., 2007). Early accumulation of JA in phloem exudates and JA-dependent gene expression in the systemic leaves of infected plants correlates with SAR, while SAR is compromised in several JA signaling mutants. However, the link between JA and SAR remains unclear since SAR is not altered in all JA signaling mutants (Cui et al., 2005; Mishina and Zeier, 2007). Accumulation of SA is required for SAR, but only in the signal-perceiving systemic tissue: grafting experiments showed that tobacco leaves infected with TMV could transmit a SAR signal despite the presence of bacterial salicylate hydroxylase ( $\mathrm{SH}$ ) encoded by the $N a h G$ gene. By contrast, expression of this SA-degrading enzyme in systemic tissue abolished SAR signal perception (Vernooij et al., 1994). In many plant species, SA undoubtedly plays an important role in both locally expressed basal resistance and SAR
(Van Loon and Antoniw, 1982).

In tobacco, injection with SA elicited enhanced resistance to TMV, and application of SA triggered the accumulation of PR-proteins (Petersen et al., 2000), leading to speculation that SA might function as the endogenous signal. NPR1 modulates cross-talk between SA and JA-dependent defense pathways. NPR1 was active by SA treatment to suppress JA-dependent defense pathways (Spoel et al., 2003). SA suppresses jasmonate-dependent gene expression. The WRKY70 transcription factor is a node of convergence for JA-mediated and SA-mediated signals in plant defense (Li et al., 2004). MAPK4 negatively regulates SAR (Petersen et al., 2000). In wild-type plants, the defence genes were activated to regulate SAR. In other defective plants which could not product SA or JA, the defense genes were also activated by treatment but the regulation of SAR was affected. These results suggested exogenous hormone must act through endogenous hormone.

Some genes were related to the antagonism of JA and SA. $P D F 1.2, P R 1$, and $P R 2$ were positive regulators of the antagonism of JA and SA. WRKY70 and NPRI were negative regulator (Shang et al., 2011). These two genes maintain an endogenous JA and SA balance in their action. We found that if the genes expression induced by exogenous JA and SA was maintained in a suitable range, it takes advantage to the plant immunity. For JA $\rightarrow$ SA, the genes induced by JA gave faster responses, and the genes induced by SA were delayed. Therefore, it can be concluded that JA may play a more important role than SA in this phytohormone interaction.

\section{Acknowledgments}

This work was supported by the National Nature Science Foundation of China (91017004, 31070210, 30970214 and 30800071) and Sichuan Nature Science Foundation (2010JQ0080), National Key Basic Research '973' Program of China (2009CB118500).

\section{References}

Cao, Y., Zhang, Z. W., Xue, L. W., Du, J. B., Shang, J., Xu, F., Yuan, S. and Lin, H. H. 2009. Lack of salicylic acid in Arabidopsis protects plants against moderate salt stress. Z. Naturforsch. 64c:231-238.

Cui, J., Bahrami, A. K., Pringle, E. G., Hernandez-Guzman, G, Bender, C. L., Pierce, N. E. and Ausubel, F. M. 2005. Pseudomonas syringae manipulates systemic plant defenses against pathogens and herbivores. Proc. Natl. Acad. Sci. USA 102: 1791-1796.

Dong, X. 2001. Genetic dissection of systemic acquired resistance. Curr. Opin. Plant Biol. 4:309-314.

Durrant, W. E. and Dong, X. 2004. Systemic acquired resistance. 
Annu. Rev. Phytopathol. 42:185-209.

Feys, B. J. and Parker, J. E. 2000. Interplay of signaling pathways in plant disease resistance. Trends Genet. 16:449-455.

Glazebrook, J., 2001. Genes controlling expression of defense responses in Arabidopsis-2001 status. Curr. Opin. Plant Biol. 4:301-308.

Hernandez, J. A., Diaz-vivancos, P., Rubio, M., Olmos, E., RosBarcelo, A. and Martinez-Gomez, P. 2006. Long-term plum pox virus infection produces an oxidative stress in a susceptible apricot, Prunus armeniaca, cultivar but not in a resistant cultivar. Physiol. Plant. 126:140-152.

Kachroo, P., Yoshioka, K., Shah, J., Dooner, H. K. and Klessig, D. F. 2000. Resistance to turnip crinkle virus in Arabidopsis is regulated by two host genes and is salicylic acid dependent but NPR1, ethylene, and jasmonate independent. Plant Cell 12: 677-690.

Li, J., Brader, G, and Palva, E. T. 2004. The WRKY70 transcription factor: anode of convergence for jasmonate mediated and salicylate2mediated2 signals in plant defense. Plant Cell 16: 319-331.

Miao, Y. and Zentgraf, U. 2007. The antagonist function of Arabidopsis WRKY53 and ESR/ESP in leaf senescence is modulated by the jasmonic and salicylic acid equilibrium. Plant Cell 19:819-830.

Mishina, T. E. and Zeier, J. 2007. Pathogen-associated molecular pattern recognition rather than development of tissue necrosis contributes to bacterial induction of systemic acquired resistance in Arabidopsis. Plant J. 50:500-513.

Petersen, M., Brodersen, P., Naested, H., Naested, H., Andreasson, E., Lindhart, U., Johansen, B., Nielsen, H. B., Lacy, M., Austin, M. J., Parker, J. E., Sharma, S. B., Klessig, D. F., Martienssen, R., Mattsson, O., Jensen, A. B. and Mundy, J. 2000. Arabidosis MAP kinase 4 negatively regulates systemic acquired resistance. Cell 103:1111-1120.

Pieterse, C. M. J., Van Wees, S. C. M., Van Pelt, J. A., Knoester, M., Laan, R., Gerrits, H., Weisbeek, P. J. and Van Loon, L. C. 1998. A novel signaling pathway controlling induced systemic resistance in Arabidopsis. Plant Cell 10:1571-1580.

Pieterse, C. M. J., Ton, J. and van Loon, L. C. 2001. Cross-talk between plant defense signaling pathway: boost or burden? Agbiotechnet 3:1-8.

Reymond, P. and Farmer, E. E. 1998. Jasmonate and salicylate as global signals for defense gene expression. Curr. Opin. Plant Biol. 1:404-411.

Spoel, S. H., Koornneef, A., Claessens, S. M. C., Korzelius, J. P., Van Pelt, J. A., Mueller, M. J., Buchala, A. J., Métraux, J. P., Brown, R., Kazan, K. Van Loon, L.C. Dong, X. N. and Pietersea, C. M. J. 2003. NPR1 modulates cross-talk between salicylate- and jasmonate-dependent defense pathways through a novel function in the cytosol. Plant Cell 15:760-770.

Spoel, S. H., Mou, Z., Tada, Y., Spivey, N., Genschik, P. and Dong, X. N. 2009. Proteasome-mediated turnover of the transcription coactivator NPR 1 plays dual roles in regulating plant immunity. Cell 137:860-872.
Shang, J., Xi, D. H., Huang, Q. R., Xu, M. Y., Yuan, S., Wang, S. D., Jia, S. D., Cao, S., Zhou, Z. L. and Lin, H. H. 2009. Effect of two satellite RNAs on Nicotiana glutinosa infected with Cucumber mosaic virus (CMV). Physiol. Mol. Plant Pathol. 74:184-190.

Shang, J., Xi, D. H., Yuan, S., Xu, F., Xu, M. Y., Qi, H. L., Wang, S. D., Huang, Q. R., Wen, L. and Lin, H. H., 2010. Difference of physiological characters in dark green islands and yellow leaf tissue of CMV-infected Nicotiana tabacum leaves. $Z$. Naturforsch. 65c:73-78.

Shang, J., Xi, D. H., Xu, F., Wang, S. D., Cao, S., Xu, M. Y., Zhao, P. P., Zhang, Z. W., Jia, S. D., Wang, J. H., Yuan, S. and Lin, H. H. 2011. A broad-spectrum, efficient and nontransgenic approach to control plant viruses by application of salicylic acid and jasmonic acid. Planta, DOI:10.1007/s00425-0101308-5.

Takahashi, H., Miller, J., Nozaki, Y., Sukamuto, Takeda, M., Shah, J., Hase, S., Ikegami, M., Ehara, Y. and Dinesh-Kumar, S. P. 2002. RCY1, an Arabidopsis thaliana RPP8/HRT family resistance gene, conferring resistance to cucumber mosaic virus requires salicylic acid, ethylene and a novel signal transduction mechanism. Plant J. 32:655-667.

Thomma, B. P. H. J., Eggermont, K., Penninckx, I. A. M. A., Mauch-Mani, B., Vogelsang, R., Cammue, B. P. A. and Broekaert, W. F. 1998. Separate jasmonate-dependent and salicylate-dependent defense-response pathways in Arabidopsis are essential for resistance to distinct microbial pathogens. Proc. Natl. Acad. Sci. USA 95:15107-15111.

Thomma, B. P. H. J., Penninckx, I. A. M. A., Cammue, B. P. A. and Broekaert, W. F. 2001. The complexity of disease signaling in Arabidopsis. Curr. Opin. Immunol. 13:63-68.

Truman, W., Bennett, M. H., Kubigsteltig, I., Turnbull, C. and Grant, M. 2007. Arabidopsis systemic immunity uses conserved defense signaling pathways and is mediated by jasmonates. Proc. Natl. Acad. Sci. USA 104:1075-1080.

Uquillas, C., Letelier, I., Blanco, F., Jordana, X. and Holuigue, L. 2004. NPR1-independent activation of immediate early salicylic acid-responsive genes in Arabidopsis. Mol. Plant-Microbe Interact. 17:34-42.

Van, Loon, L. C. and Antoniw, J. F. 1982. Comparison of the effects of salicylic acid and ethephon with virus-induced hypersensitivity and acquired resistance in tobacco. Net. J. Plant Pathol. 88:237-256.

Velikova, V., Yordanov, I. and Edreva, A. 2000. Oxidative stress and some antioxidant system in acid rain-treated bean plants. Protective role of exogenous polymines. Plant Sci. 151:59-66.

Vernooij B., Friedrich, L., Morse, A., Reist, R., Kolditz-Jawhar, R., Ward, E., Uknes, S., Kessmann, H. and Ryals, J. 1994. Salicylic acid is not the translocated signal responsible for inducing systemic acquired resistance but is required in signal transduction. Plant Cell 6:959-965.

Xi, D. H., Feng, H., Lan, L. Q. Du, J. B., Wang, J. H., Zhang, Z. W., Xue, L. W., Xu, W. and Lin, H. H. 2007. Characterization of synergy between cucumber mosaic virus and tobacco necrosis virus in Nicotiana benthamiana. J. Phytopath. 155:570-573. 